\title{
Partitioning of Charge in Dibenzo[b,g]chrysene: The Role of the System's Topology
}

\author{
Lior Zelikovich, Roy E. Hoffman, Ari Ayalon and Mordecai Rabinovitz* \\ The Hebrew University of Jerusalem, Jerusalem 91904, Israel
}

\begin{abstract}
Dibenzo $[b, g]$ chrysene dianion $\left(3^{2-}\right)$ shows in its NMR spectrum a partitioning of charge. In the dianion most of the charge is located on the 'anthracene' moiety. These observations have been confirmed by MNDO calculations. In the calculations, the location of the lithium cation is taken into account. This unusual phenomenon is connected with the system's paratropicity and with its charge alternation. The NMR assignments were assisted by 2D techniques.
\end{abstract}

The study of charged polycyclic systems has twofold aspects: on the one hand they can serve as model compounds for electronic theories and, on the other ${ }^{1-5}$ as reactive intermediates. An interesting and little explored group of carbanions is the series of $\pi$-conjugated anions, more specifically, the $4 n \pi$-conjugated dianions, ${ }^{6}$ entitled paratropic antiaromatic. This class of anions shows an unexpected stability and they are prepared by two consecutive electron reduction processes from the parent neutral $(4 n+2) \pi$ system. The polycyclic $4 n \pi$ anions are therefore ideal models for the study of paratropic systems, mainly by NMR spectroscopy ${ }^{7}$ with respect to their magnetic properties as well as their mode and pattern of electron delocalization. Extensive NMR studies revealed some fundamental properties of these charged systems. ${ }^{7}$ The charged systems are characterized by a narrow LUMO-HOMO gap $(\Delta E)$ which serves as the parameter which governs both their paratropicity (high-field shift) and their ${ }^{1} \mathrm{H}$ NMR line shape. ${ }^{8,9}$ A correlation has been suggested between the experimental criterion for paratropicity and the theoretical notion of antiaromaticity in $4 n \pi$ polycyclic, carbocyclic and heterocyclic dianions. ${ }^{10,11}$ This correlation is based on $\Delta E$ of the system which, in turn, is governed by the system's topology, symmetry, and geometry. ${ }^{12,13}$ Structural deviations from the symmetrical triphenylene dianion towards less symmetrical systems, twisted systems, ${ }^{13}$ or the introduction of a heteroatom, ${ }^{12}$ e.g. nitrogen, reduce the efficiency of the electron delocalization followed by a concomitant quenching of paratropicity. Dianions of annulenoannulenes have recently been prepared and used to distinguish between different modes of $\pi$-delocalization patterns. ${ }^{14,15}$ Those of the simple benzenoid hydrocarbons exhibit the expected even $\pi$-charge distribution over the entire carbon framework with an alternating mode along the perimeter. ${ }^{15}$

It was therefore surprising to find by ${ }^{1} \mathrm{H}$ and ${ }^{13} \mathrm{C}$ NMR studies that the dianion of dibenzo[a,c]naphthacene (1), i.e. $\mathbf{1}^{2-}$, behaves as if it were composed of two separate substructures: a linear and an angular one, or 'anthracene' and 'phenanthrene' components, thus indicating a segregation within the pertinent antibonding orbital. ${ }^{16}$ This phenomenon was also observed in the dianion derived from the heterocyclic analogue of $\mathbf{1}$, i.e. tribenzo[ $a, c, i]$ phenazine (2). It seems as if the system's topology dictates the pattern of electron delocation. The $\pi$ spin distribution of $1^{-}$also reflected the $\pi$-charge distribution of $1^{2-} .^{16}$

In view of the unusual charge distribution pattern of $\mathbf{1}^{2-}$ in which the 'anthracene' moiety seems to bear most of the charge, it was decided to study dibenzo $[b, g]$ chrysene dianion, $\mathbf{3}^{2-}$, which is also composed of an 'anthracene' and a 'phenanthrene' or a 'biphenyl' moiety and is an isomer of $\mathbf{1}^{2-}$. System $3^{2-}$ has a different symmetry than that of $\mathbf{1}^{2-}$ but can still be viewed as being composed of a linear and an angular component.
Dibenzo $[b, g]$ chrysene (3) was prepared and underwent a two-stop electron addition process with alkali metals. 2D NMR techniques afforded an unequivocal assignment of protons and carbons and the chemical shift differences of each nucleus between the neutral and the charged system afforded the local charge densities on the carbons (vide infra). MNDO calculations, which took into account the location of the lithium cations, afforded the calculated charge densities which were compared with the experimental data. The charge delocalization pattern and its relationship to the location of the countercation as well as the degree of paratropicity of the system and its dependence on temperature and countercation afforded a better understanding of this unusual phenomenon.



\section{Results}

The parent hydrocarbon 3 was prepared by UV irradiation of 1-(2-naphthyl)-2-(9-phenanthryl)ethene (4). The latter being prepared by a modified literature procedure, ${ }^{17}$ via the Wittig reaction between phenanthrene-9-carbaldehyde and the phosphonium salt prepared from 2-bromomethylnaphthalene.

The proton and carbon spectra ${ }^{17}$ of 3 which appear in Fig. 1, were assigned with the aid of 2D NMR techniques. Proton and carbon assignments appear in Tables 1 and 2, respectively. When the solution of 3 in $\left(\left[{ }^{2} \mathrm{H}_{8}\right] \mathrm{THF}\right)$ was brought into contact with the alkali metal ( $\mathrm{Li}$ or $\mathrm{Na}$ ), a dark red colour appeared after several days but no ${ }^{1} \mathrm{H}$ NMR spectrum could be detected in the temperature range $200-300 \mathrm{~K}$. The solution turned dark blue after $c a$. one week and an NMR spectrum of the dianion $\mathbf{3}^{2-}$ (protons and carbons) could be observed (Fig. 2 and Tables 1 and 2). The full assignment was carried out by TOCSY, NOESY (Fig. 3) one bond $\mathrm{C}-\mathrm{H}$, and by $\mathrm{LR} \mathrm{C}-\mathrm{H}$ correlation experiments. There are two distinct groups of absorptions in the ${ }^{1} \mathrm{H}$ NMR spectrum of $3^{2-}$ : one in the region $6.8-5.5 \mathrm{ppm}$ and 

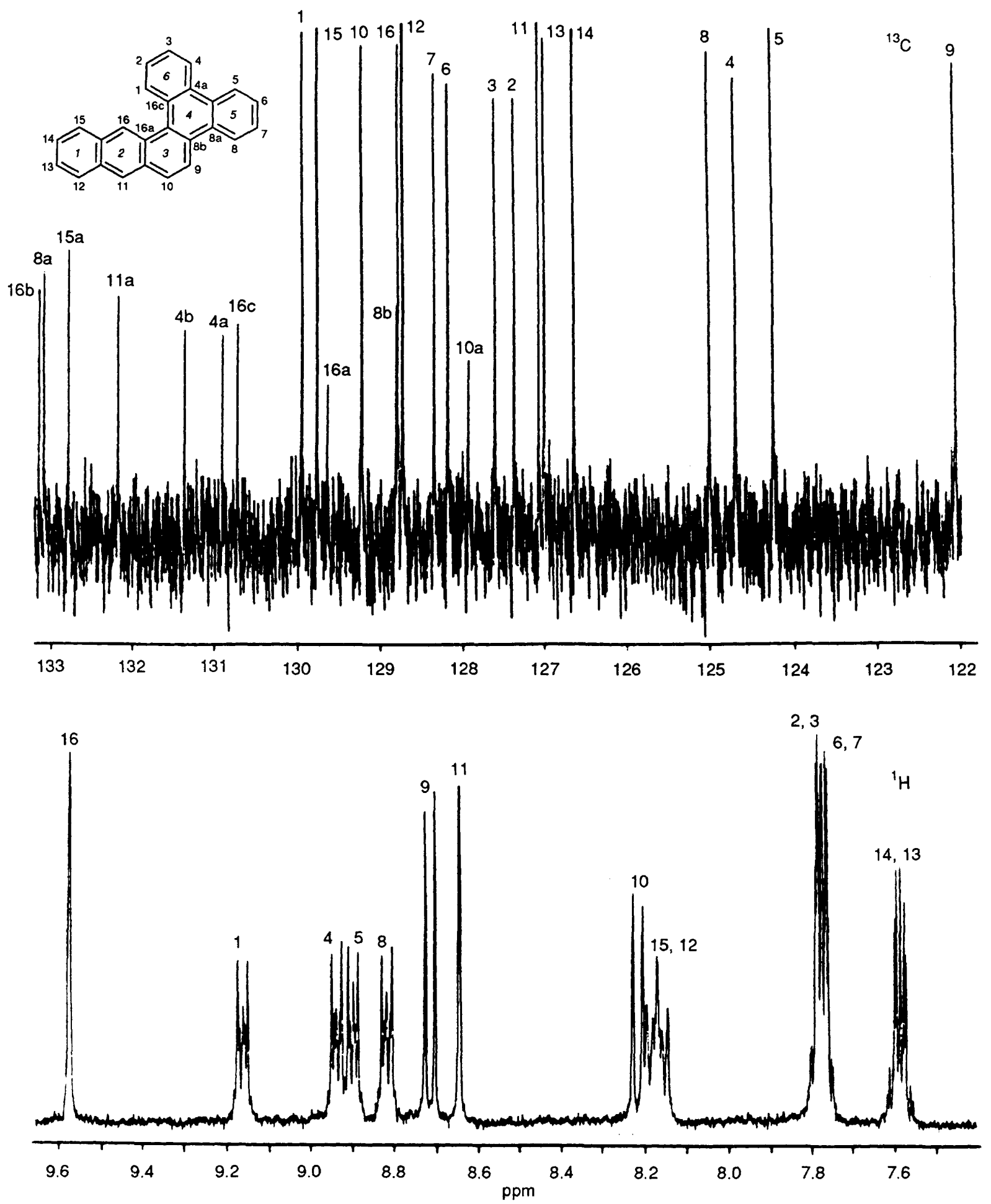

Fig. $1{ }^{1} \mathrm{H}$ and ${ }^{13} \mathrm{C}$ NMR spectra of 3 , in $\left[{ }^{2} \mathrm{H}_{8}\right] \mathrm{THF}$, chemical shifts relative to TMS

the other in the range $4.5-1.8 \mathrm{ppm}$. The TOCSY spectrum made possible differentiation between the spin systems where each spin system resides on a different ring. The NOESY experiments enabled the differentiation of protons on 'bay' regions or at peri positions and thus afford the connectivities between the various spin systems (Fig. 3).

The doublet at $3.80 \mathrm{ppm}$ is only correlated in the TOCSY spectrum to the doublet at $2.40 \mathrm{ppm}$ and the two doublets therefore represent protons $9-\mathrm{H}$ and $10-\mathrm{H}$, respectively. From the NOESY spectrum it can be seen that 9-H interacts with 8-H. One can therefore follow the connectivities in ring 5 which bears $8-\mathrm{H}$ and from the TOCSY spectrum protons $5-\mathrm{H}(\mathrm{d})$,
6-H(t), 7-H(t) and 8-H(d) can be assigned. From the NOESY spectrum (Fig. 3) it can be shown that the absorption at 6.10 $\mathrm{ppm}$ interacts with $5-\mathrm{H}$ and is therefore assigned to $4-\mathrm{H}$. The TOCSY spectrum affords the assignment of $1-\mathrm{H}(\mathrm{d}), 2-\mathrm{H}(\mathrm{t})$ and $3-\mathrm{H}(\mathrm{t})$ of ring 6 . Proton $1-\mathrm{H}$ of ring 6 correlates in the NOESY spectrum with $16-\mathrm{H}(\mathrm{s})$ and $16-\mathrm{H}$ correlates with $15-\mathrm{H}(\mathrm{d})$. From the TOCSY spectrum one can therefore assign $12-\mathrm{H}(\mathrm{d}), 13-\mathrm{H}(\mathrm{t})$ and $14-\mathrm{H}(\mathrm{t})$ on ring 1 . The singlet of $11-\mathrm{H}$ also shows a NOESY connectivity with $10-\mathrm{H}$. From all the above we could complete the assignment of the proton spectrum of $3^{2-} / 2 \mathrm{Na}^{+}$.

We performed two ${ }^{1} \mathrm{H}_{-}{ }^{13} \mathrm{C} 2 \mathrm{D}$ correlation experiments. The one bond heteronuclear correlation experiment was optimized 
Table $1{ }^{1} \mathrm{H}$ NMR chemical shifts of 3 and $3^{2-a}$

\begin{tabular}{|c|c|c|c|c|c|c|c|c|c|c|c|c|c|c|c|c|c|c|c|}
\hline \multirow[b]{2}{*}{ Structure } & \multirow[b]{2}{*}{$T / \mathbf{K}$} & \multirow[b]{2}{*}{ Counterion } & \multicolumn{16}{|c|}{ Proton } & \multirow{2}{*}{$\begin{array}{l}\text { Centre } \\
\text { of } \\
\text { gravity }\end{array}$} \\
\hline & & & 1 & 2 & 3 & 4 & 5 & 6 & 7 & 8 & 9 & 10 & 11 & 12 & 13 & 14 & 15 & 16 & \\
\hline 3 & 295 & & 9.17 & 7.77 & 7.77 & 8.94 & 8.91 & 7.77 & 7.77 & 8.81 & 8.70 & 8.22 & 8.64 & 8.14 & 7.59 & 7.60 & 8.17 & 9.58 & 8.34 \\
\hline $3^{2-}$ & 300 & $\mathrm{Li}^{+}$ & 6.6 & 5.7 & 5.4 & 5.9 & 6.1 & 5.7 & 6.0 & 5.7 & 3.77 & 2.40 & 2.08 & 3.60 & 4.51 & 4.31 & 3.66 & 3.20 & 4.66 \\
\hline $3^{2-}$ & 273 & $\mathrm{Li}^{+}$ & 6.44 & 5.57 & 5.21 & 5.76 & 5.94 & 5.57 & 5.76 & 5.51 & 3.64 & 2.30 & 1.96 & 3.48 & 4.53 & 4.28 & 3.57 & 3.11 & 4.53 \\
\hline $3^{2-}$ & 200 & $\mathrm{Li}^{+}$ & 6.01 & 5.28 & 4.88 & 5.28 & 5.53 & 5.28 & 5.53 & 5.12 & 3.30 & 2.07 & 1.67 & 3.15 & 4.15 & 3.97 & 3.24 & 2.85 & 4.20 \\
\hline $3^{2-}$ & 300 & $\mathrm{Na}^{+}$ & 6.70 & 5.81 & 5.53 & 6.10 & 6.28 & 5.83 & 5.98 & 5.73 & 3.80 & 2.40 & 1.92 & 3.41 & 4.33 & 4.25 & 3.60 & 3.10 & 4.67 \\
\hline $3^{2-}$ & 273 & $\mathrm{Na}^{+}$ & 6.53 & 5.71 & 5.42 & 5.98 & 6.16 & 5.75 & 5.90 & 5.66 & 3.71 & 2.36 & 1.90 & 3.39 & 4.30 & 4.23 & 3.58 & 3.08 & 4.60 \\
\hline $3^{2-}$ & 200 & $\mathrm{Na}^{+}$ & 6.42 & 5.54 & 5.24 & 5.78 & 5.95 & 5.58 & 5.74 & 5.43 & 3.54 & 2.21 & 1.70 & 3.30 & 4.18 & 4.00 & 3.30 & 2.90 & 4.41 \\
\hline
\end{tabular}

${ }^{a}$ In $\left[{ }^{2} \mathrm{H}_{8}\right] \mathrm{THF}$ in ppm relative to TMS; experimental error $\pm 0.01 \mathrm{ppm}$.


Fig. $2{ }^{1} \mathrm{H}$ and ${ }^{13} \mathrm{C}$ NMR spectra of $3^{2-} / 2 \mathrm{Na}^{+}$at $295 \mathrm{~K}$, in $\left[{ }^{2} \mathrm{H}_{8}\right] \mathrm{THF}$, chemical shifts relative to TMS

for ${ }^{1} J_{\mathrm{C}-\mathrm{H}}=160 \mathrm{~Hz}$ and afforded the assignment of the carbons which are directly attached to protons $\mathrm{C}-1$ through $\mathrm{C}-16$. The Long Range experiment was optimized for ${ }^{3} J_{\mathrm{C}-\mathrm{H}}=12 \mathrm{~Hz}$ and thus afforded the long-range coupling between protons and quaternary carbons.

The NMR experiments were performed in the temperature range $200-300 \mathrm{~K}$ in $\left[{ }^{2} \mathrm{H}_{8}\right] \mathrm{THF}$ solution with $\mathrm{Li}^{+}$and $\mathrm{Na}^{+}$as counter cations. The proton and carbon chemical shifts appear in Tables 1 and 2 . In the case of $3^{2-} / 2 \mathrm{Li}^{+}$the line shape of one part of the spectrum is temperature dependent (Fig. 4, vide infra). In the case of $3^{2-} / 2 \mathrm{Na}^{+}$only slight changes of the chemical shifts could be observed. In order to make sure that no skeletal changes had occurred, each sample of the dianions was quenched with oxygen. Only the starting material, i.e. 3, was subsequently detected by ${ }^{1} \mathrm{H}$ NMR spectroscopy.

\section{Discussion}

In order to understand clearly the mode of delocalization and charge distribution of the anion $3^{2-}$, we estimated the local charge density on each carbon atom. The experimental estimation was carried out using chemical shift-charge density correlations. ${ }^{18}$ As carbon atoms are not sensitive to anisotropic effects, their chemical shifts are a reliable probe for the charge density residing on them (vide infra). Eqn. (1) gives the charge

$$
\Delta q_{\mathrm{c}_{\mathrm{i}}}=K_{\mathrm{c}} \Delta \delta_{\mathrm{c}_{\mathrm{i}}}
$$

density on each particular ${ }^{13} \mathrm{C}$ nucleus, where $\Delta q_{\mathrm{c}_{\mathrm{i}}}$ is the additional charge density as compared with the neutral species; $\Delta \delta_{\mathrm{c}}$ is the change of the chemical shift of the anion, as compared with the shift of the relevant carbon atom in the neutral hydrocarbon, given by eqn. (2); and $K_{\mathrm{c}}$ is a correlation

$$
\Delta \delta_{\mathrm{c}_{\mathrm{i}}}=\delta_{\mathrm{c}_{\mathrm{i}}}(3)-\delta_{\mathrm{c}_{\mathrm{i}}}\left(3^{2-}\right)
$$

constant of the charge-chemical shift correlation. It should be noted that the value of $K_{\mathrm{c}}$ is characteristic of the family of anions under consideration. It can be estimated by the method of Müllen and Edlund ${ }^{18}$ and was calculated to be, in our case $71 \mathrm{ppm} / \mathrm{e}$. Another way to calculate $K_{\mathrm{c}}$ is to divide the total change of chemical shifts of the carbons, upon charging, by the number of charges (in this case two) in the anionic system. 
Table $2{ }^{13} \mathrm{C}$ Chemical shifts (ppm) and charge densities of $3^{2-a}$

\begin{tabular}{|c|c|c|c|c|c|c|c|c|c|c|c|c|c|c|}
\hline \multirow[b]{2}{*}{ System } & \multirow[b]{2}{*}{$T / K$} & \multirow[b]{2}{*}{ Counterion } & \multicolumn{12}{|c|}{ Carbon } \\
\hline & & & 1 & 2 & 3 & 4 & 5 & 6 & 7 & 8 & 9 & 10 & 11 & 12 \\
\hline $3^{b}$ & 295 & & 129.91 & 127.35 & 127.59 & 124.67 & 124.22 & 128.34 & 128.17 & 124.97 & 122.00 & 129.21 & 127.05 & 128.72 \\
\hline $3^{2-c}$ & $273^{\circ}$ & $\mathrm{Li}^{+}$ & 116.1 & 126.5 & 115.0 & 122.0 & 121.7 & 119.6 & 126.5 & 116.9 & 111.1 & 96.0 & 91.6 & 111.1 \\
\hline $3^{2-b}$ & 200 & $\mathrm{Li}^{+}$ & 115.4 & 126.00 & 114.21 & 121.82 & 121.50 & 119.45 & 127.00 & 116.35 & 111.30 & 97.20 & 92.35 & 111.26 \\
\hline $3^{2-b}$ & 300 & $\mathrm{Na}^{+}$ & 116.90 & 126.40 & 116.65 & 122.24 & 121.93 & 120.34 & 126.90 & 117.10 & 111.36 & 95.75 & 88.85 & 110.90 \\
\hline $3^{2-b}$ & 273 & $\mathrm{Na}^{+}$ & 116.23 & 126.35 & 116.34 & 122.20 & 121.83 & 120.26 & 126.85 & 117.00 & 111.28 & 95.90 & 89.54 & 111.00 \\
\hline $3^{2-b}$ & 200 & $\mathrm{Na}^{+}$ & 115.70 & 126.20 & 115.39 & 121.90 & 121.65 & 119.55 & 126.80 & 116.64 & 111.22 & 97.15 & 90.74 & 111.54 \\
\hline $3^{2-d}$ & & $\mathrm{Li}^{+}$ & -0.17 & -0.01 & -0.15 & -0.03 & -0.03 & -0.10 & -0.01 & -0.10 & -0.14 & -0.45 & -0.52 & -0.24 \\
\hline $3^{2-e}$ & & $\mathrm{Li}^{+}$ & -0.14 & -0.07 & -0.14 & -0.05 & -0.09 & -0.10 & -0.08 & -0.09 & -0.19 & -0.40 & -0.44 & -0.10 \\
\hline
\end{tabular}

${ }^{a}$ In $\left[{ }^{2} \mathrm{H}_{8}\right]$ THF relative to TMS, experimental error $\pm 0.01 \mathrm{ppm} .{ }^{b}$ NMR carbon chemical shifts. ${ }^{c}$ Lines are too broad beyond this temperature.

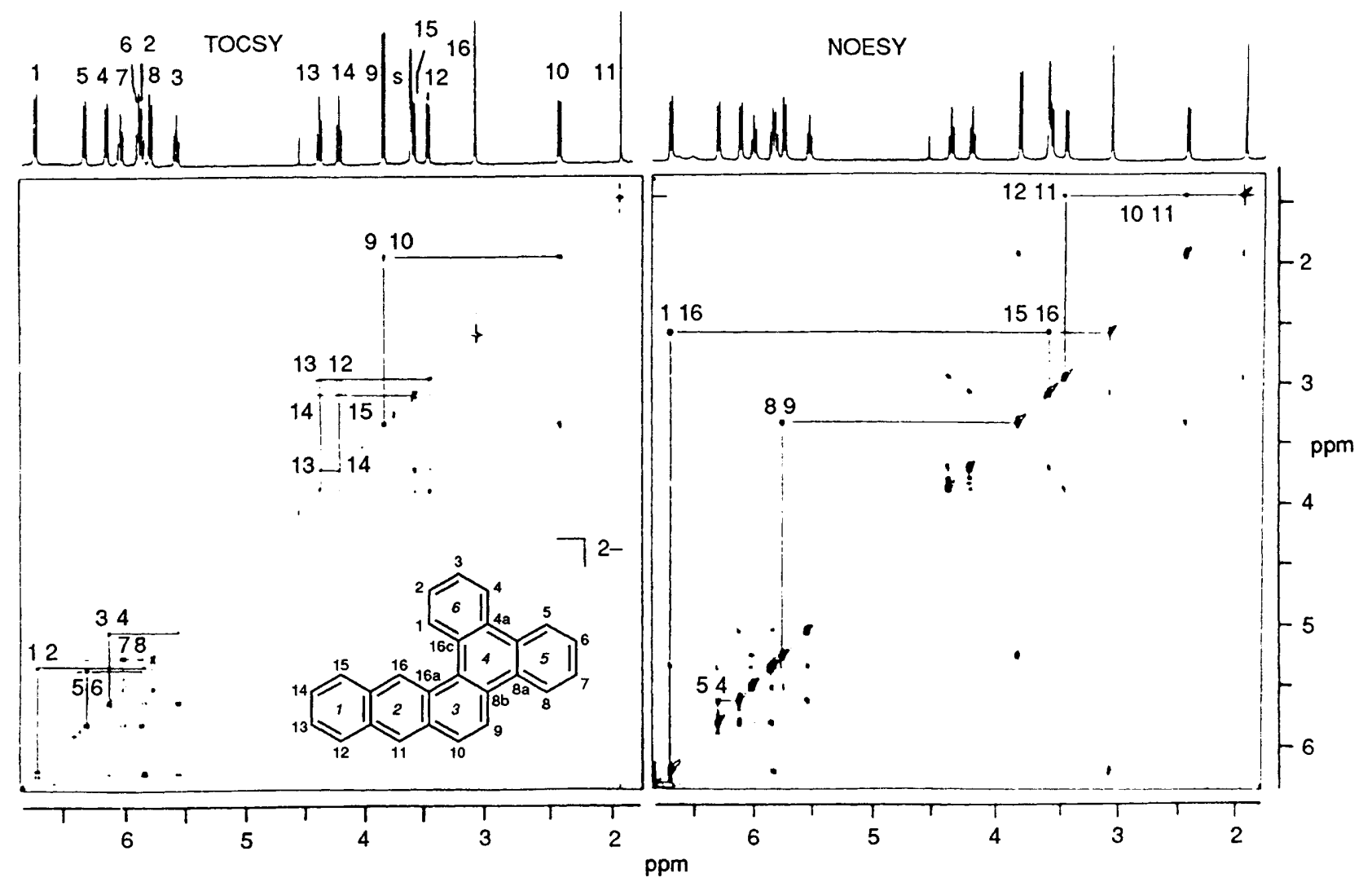

Fig. 3 TOCSY and NOESY 2D NMR spectra of $3^{2-} / 2 \mathrm{Na}^{+}$, in $\left[{ }^{2} \mathrm{H}_{8}\right] \mathrm{THF}$ at $295 \mathrm{~K}$

From the latter calculation we get $K_{\mathrm{c}}=72.8 \mathrm{ppm} / \mathrm{e}$. This value was used in the present study. The additional charge densities due to the charging process are given in Table 2.

Fig. 5 shows the charge densities as obtained from the ${ }^{13} \mathrm{C}$ NMR experiments. It clearly shows that the majority of the charge is located on the 'anthracene-like' moiety of $3^{2-}$, i.e. on carbons $8 \mathrm{~b}$ to $16 \mathrm{~b}$. In this part of the anion, the charge is located in an alternating fashion. It can be seen that the 'anthracene' moiety has a symmetry axis perpendicular to the bonds connecting $\mathrm{C}-13$ to $\mathrm{C}-14$ and $\mathrm{C}-9$ to $\mathrm{C}-8 \mathrm{~b}$ (Fig. 5). The additional charge density on $\mathrm{C}-11$ is similar to that of $\mathrm{C}-16$. The other component of $\mathbf{3}^{2-}$ which can be referred to as the 'biphenyl-like' or 'phenanthrene-like' moiety has hardly any negative charge. This component includes $\mathrm{C}-1$ to $\mathrm{C}-8 \mathrm{a}$ and $\mathrm{C}-16 \mathrm{c}$. The charge here shows a lower degree of alternation. However, even the minor additional charge density on this component is spread symmetrically along an axis which is perpendicular to the C-4a-C-4b bond (Fig. 5). This observation of partitioning of charge is very similar to the pattern of charge delocalization which was observed in $\mathbf{1}^{2-} .{ }^{16}$ Systems $\mathbf{1}^{2-}$ and $\mathbf{3}^{2-}$ have different symmetries, but despite this difference, both of them maintain the same pattern of charge segregation. The experimental charge densities were compared with the calculated ones.

Calculations were performed while taking into account the position of the countercation, in our case the lithium ion. We considered the structural features of dibenzo $[b, g]$ chrysene dilithium salt, i.e. $3^{2-} / 2 \mathrm{Li}^{+}$by exploring its multidimensional potential energy surface. We relied extensively on Dewar's MNDO method ${ }^{19}$ with Thiel's lithium parameters. ${ }^{20}$ All internal degrees of freedom were relaxed during the geometry optimization unless otherwise noted. All molecules were considered to exist as ground state singlets. MNDO is known to be an especially useful method for predicting the structures of mono- and di-lithiated hydrocarbons. ${ }^{21}$

We explored 15 structures of $3^{2-} / 2 \mathrm{Li}^{+}$and for each of them the geometry was optimized so that the local minimum energy $\left(\Delta H_{\mathrm{f}}^{\circ}\right)$ was obtained. This geometry depends on the initial 


\begin{tabular}{lllllllllllllll}
\hline 13 & 14 & 15 & 16 & $4 \mathrm{a}$ & $4 \mathrm{~b}$ & $8 \mathrm{a}$ & $8 \mathrm{~b}$ & $10 \mathrm{a}$ & $11 \mathrm{a}$ & $15 \mathrm{a}$ & $16 \mathrm{a}$ & $16 \mathrm{~b}$ & $16 \mathrm{c}$ & Centre of gravity \\
\hline 126.97 & 126.40 & 129.73 & 128.75 & 130.90 & 131.40 & 133.10 & 128.70 & 127.90 & 132.20 & 132.70 & 129.60 & 133.20 & 130.76 & 128.57 \\
120.7 & 116.9 & 113.0 & 91.6 & 136.1 & 135.6 & 141.7 & 122.0 & 157.8 & 149.8 & 148.3 & 147.5 & 103.0 & 147.4 & 123.32 \\
120.32 & 116.40 & 112.43 & 91.96 & 137.01 & 135.93 & 142.52 & 122.50 & 157.72 & 150.16 & 149.08 & 148.62 & 103.87 & 148.10 & 123.41 \\
121.60 & 117.70 & 113.00 & 90.60 & 135.70 & 134.35 & 140.00 & 122.26 & 155.75 & 149.47 & 148.63 & 147.10 & 100.80 & 145.80 & 123.00 \\
121.45 & 117.50 & 112.95 & 91.00 & 135.90 & 134.76 & 140.26 & 122.37 & 155.93 & 149.55 & 148.85 & 147.39 & 101.15 & 145.90 & 123.08 \\
121.10 & 116.94 & 112.91 & 91.57 & 136.49 & 135.18 & 141.22 & 122.43 & 156.40 & 149.78 & 149.12 & 147.66 & 102.63 & 147.06 & 123.20 \\
-0.07 & -0.12 & -0.23 & -0.52 & +0.06 & +0.04 & +0.10 & -0.08 & +0.30 & +0.20 & +0.20 & +0.25 & -0.44 & +0.20 & \\
-0.09 & -0.13 & -0.08 & -0.38 & -0.05 & +0.02 & -0.02 & -0.08 & +0.03 & -0.06 & -0.01 & +0.02 & -0.44 & +0.11 & \\
\hline
\end{tabular}

${ }^{d}$ Charge densities as deduced from ${ }^{13} \mathrm{C}$ NMR. ${ }^{e}$ Charge densities as deduced from MNDO calculations for structure A (Table 3).



Fig. 4 Line broadening in the ${ }^{1} \mathrm{H}$ NMR spectra of $3^{2-} / 2 \mathrm{Li}^{+}$ (temperature range $200-300 \mathrm{~K},\left[{ }^{2} \mathbf{H}_{8}\right] \mathrm{THF}$ )

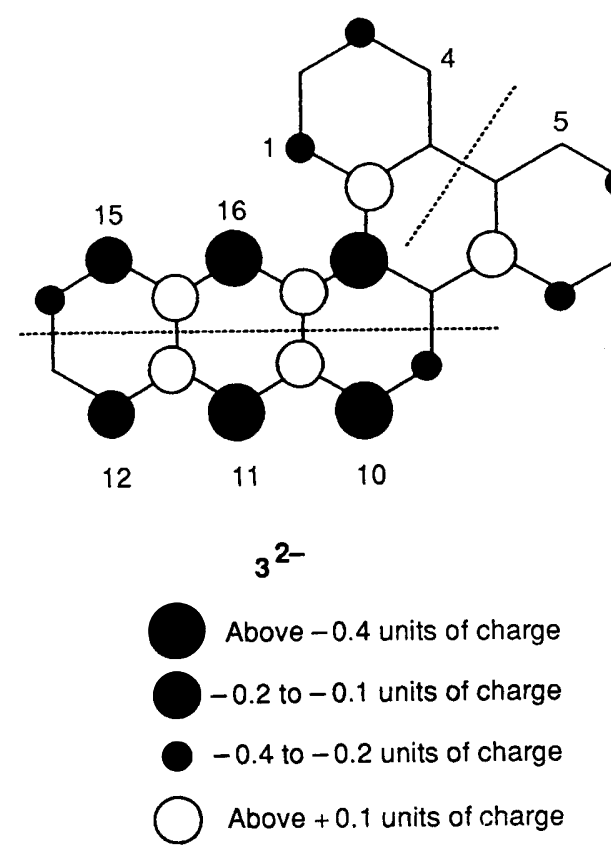

Fig. 5 Experimental charge distribution of $3^{2-}\left({ }^{13} \mathrm{C}\right.$ NMR). Solid circles represent negative charge concentration, open circles represent positive charge localization.
Table 3 Heats of formation (MNDO) of $3^{2-} / 2 \mathrm{Li}^{+}$as a function of counter ion position

\begin{tabular}{lll}
\hline Structure & $\begin{array}{l}\text { Location of lithium } \\
\text { cation above and } \\
\text { below rings }\end{array}$ & $\begin{array}{l}\Delta H_{\mathrm{f}}^{\circ} / \\
\mathrm{kcal} \mathrm{mol}^{-1}\end{array}$ \\
\hline $\mathrm{O}$ & 1,5 & 89.25 \\
$\mathrm{~N}$ & 1,4 & 88.01 \\
$\mathrm{M}$ & 1,6 & 87.34 \\
$\mathrm{~L}$ & 2,5 & 86.70 \\
$\mathrm{~K}$ & 4,6 & 86.61 \\
$\mathrm{~J}$ & 5,6 & 86.51 \\
$\mathrm{I}$ & 2,6 & 85.39 \\
$\mathrm{H}$ & 2,4 & 83.86 \\
$\mathrm{G}$ & 3,5 & 81.83 \\
$\mathrm{~F}$ & 4,5 & 81.26 \\
$\mathrm{E}$ & 3,4 & 78.35 \\
$\mathrm{D}$ & 1,3 & 77.61 \\
$\mathrm{C}$ & 3,6 & 76.99 \\
$\mathrm{~B}$ & 1,2 & 75.80 \\
$\mathrm{~A}$ & 2,3 & 74.59 \\
\hline
\end{tabular}

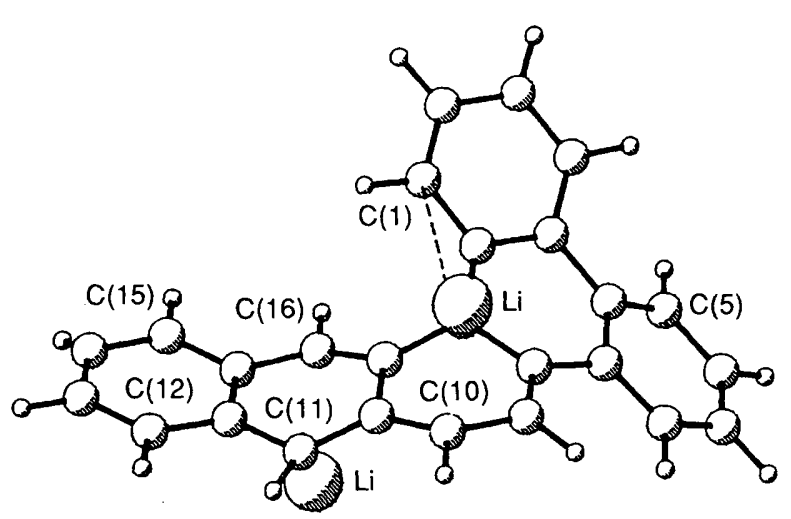

Fig. 6 Spatial structure of the optical structure of $3^{2-} / 2 \mathrm{Li}^{+}$(MNDO calculations)

geometry of each structure, and it was always close to the initial geometry. All structures which were considered had each lithium ion initially located above or below the molecular plane at two different rings. We limited ourselves to these structures as it was previously shown that lithiated polycyclic systems have an energetically preferred structure when the two countercations are located above and below the average molecular plane. ${ }^{22}$

The calculated heats of formation $\left(\Delta H_{\mathrm{f}}^{\circ}\right)$ as a function of the location of the lithium cations are reported in Table 3. Structure A in Table 3 (Fig. 6) represents a global energy minimum and its calculated charge densities on the various carbon atoms are shown in Table 2 . It is apparent that there is a good agreement between the calculated and the experimental 
$\left({ }^{13} \mathrm{C}\right.$ NMR) charge densities (Table 2). Calculations show that most of the charge is located on the 'anthracene' moiety especially on carbon atoms C-10, C-11, C-16 and C-16b. Charge alternation can also be observed although this phenomenon is more pronounced in the experimental NMR data. As expected, the rings to which the lithium atoms are attached (rings 2 and 3 ) are the rings upon which most of the charge is concentrated, in the most stable structure A (Table 2). The calculated charge density at C-12 and C-15 (Table 2) deviates somewhat from the experimental data. The ${ }^{13} \mathrm{C}$ spectrum shows that these carbons bear a higher charge density than that calculated for the optimized structure. This deviation is not surprising as in reality one can consider an equilibrium between the optimal structures where the lithium atoms are linked to rings 2 and 3 and a structure where the lithium atoms are linked to rings 1 and 2 (structures A and B in Table 3). The latter is calculated to be the next energetically preferred and only deviates slightly from the former. Therefore, an equilibrium which still favours structure A may exist. The contribution calculated from the energy difference of structure B varies between $5 \%$ at low temperature $(200 \mathrm{~K})$ and $12 \%$ at $300 \mathrm{~K}$. As will be discussed later, the increase of the population of structure $\mathrm{B}$ as a function of elevation of the temperature becomes insignificant owing to dominant ion-solvation effects. Due to a contribution from structure B some negative charge may be induced also on ring 1 . However, the placement of one of the cations above ring 3 is, in any case, preferred to its placement on ring 1 . It can also be observed that a cation adjacent to ring 3 would interact with carbon atom C-1 (ring 6) which is located inside the 'bay' of the twisted molecule (Fig. 6). Such an interaction is deduced from the higher charge density at $\mathrm{C}-1$, higher than the charge density on the other carbons of the 'biphenyl' moiety as obtained from both experiment and calculations.

As explained above, the two cations are located on adjacent rings above and below the average plane in the 'anthracene' moiety. Rabideau has shown that this is also the situation in dilithiated anthracene and phenanthrene. ${ }^{22}$ Although intuition may lead to the conclusion that owing to charge repulsion the lithium atoms should be placed as far apart as possible calculations predict that structures $\mathrm{G}-\mathrm{O}$ (Table 3 ) have high $\Delta H_{\mathrm{f}}{ }^{\circ}$ values. The rationale for this observation comes from the fact that each lithium cation can interact not only with the carbons of the ring on which it is located, but also with the negatively charged carbons of the adjacent ring, while the mutual interaction between the cations is diminished and screened by the carbon skeleton (Fig. 6). The question now arises as to why the anthracene moiety should bear most of the charge. It has been previously shown ${ }^{8}$ that anthracene is less paratropic than phenanthrene. The situation in $3^{2-}$ can be viewed as if the system tends to minimize paratropicity (antiaromaticity) by bisecting itself into two systems: the highly charged system is the less paratropic one, and the less charged system represents the neutral, more stable system. This explanation results from the segregation of the MOs. There is also a second factor which helps to stabilize the charge on the 'anthracene' moiety. Stabilization of charged systems can also be increased by alternation of charge,${ }^{15}$ which is most efficient in the 'anthracene' moiety owing to the alternate arrangement of quaternary and hydrogen-bearing carbon atoms. The latter bear negative charges more efficiently while the quaternary carbons can even be positive (Fig. 5). The highly charged carbons, e.g. C-10, C-11, C-16 and C-16b, are separated by positively charged carbons $\mathrm{C}-10 \mathrm{a}$ and $\mathrm{C}-16 \mathrm{~d}$. From all the above it can be concluded that it is not surprising that one part of the molecule will tend to stabilize a charge on account of the other. This situation leads to the formation of two moieties with unequal charge concentration leading to: $(a)$ segregation of charge, $(b)$ selective temperature-dependent line-shape of the
Table 4 Variation of the HOMO-LUMO separation $(\Delta E)$ as a function of the distance ${ }^{a}$ of the counter cation from the carbon skeleton of $3^{2-} / 2 \mathrm{Li}^{+}$

\begin{tabular}{ll}
\hline $\begin{array}{l}\text { Distance } \mathrm{Li}^{+} \text {removed } \\
\text { from optimum } \\
\text { structure }\end{array}$ & $\Delta E / \mathrm{eV}$ \\
\hline 0 & 5.83 \\
0.1 & 5.79 \\
0.2 & 5.74 \\
0.3 & 5.67 \\
0.5 & 5.56 \\
\hline
\end{tabular}

${ }^{a}$ From optimized structure $\mathrm{A}$ in Table $3{ }^{b}$ From centre of rings 2 and 3 , distance in $\AA$.

lithium salt (Fig. 4), and (c) symmetrical charge distribution (Fig. 5) (vide infra). Similar phenomena have also been observed in dibenzo $[a, c]$ naphthacene dianion $\mathbf{1}^{2-}$. The behaviour of $1^{2-} / 2 \mathrm{Li}^{+}$should be noted as it shows a temperature-dependent spectrum. This observation is rationalized ${ }^{23}$ by the larger LUMO coefficients of the 'phenanthrene' than those of the 'anthracene' moiety. It seems that the observed line shape of $3^{2-} / 2 \mathrm{Li}^{+}$originates from the same rationale. A higher triplet spin density in the 'biphenyl' moiety is expected in those carbons which have a larger LUMO coefficient. Taking into account the very narrow HOMO-LUMO gap $(\Delta E)$ such a thermal promotion of electrons can occur.

Influence of Temperature and Paratropicity on Charge Distribution.-Apart from the temperature-dependent lineshape of $3^{2-} / 2 \mathrm{Li}^{+}$arising from the thermal excitation of an electron, one can observe a temperature dependence of the chemical shifts (Tables 1 and 2). The observed shifts are displaced by up to $0.5 \mathrm{ppm}$ in the proton signals and $1.5 \mathrm{ppm}$ in the ${ }^{13} \mathrm{C}$ NMR spectrum. These changes are small but consistent. Upon elevation of the temperature all protons and most carbon absorptions are shifted to low field. However, the resonances of $\mathrm{C}-10, \mathrm{C}-11, \mathrm{C}-16$ and $\mathrm{C}-16 \mathrm{~b}$ are shifted to high field. There is almost no change in the mean carbon chemical shift (Table 2). The chemical shifts of $3^{2-} / \mathrm{Li}^{+}$at higher temperature resemble those of $3^{2-} / 2 \mathrm{Na}^{+}$at low temperature, owing to ion-solvation equilibrium. We rationalize these observations for $3^{2-}$ in terms of the formation at higher temperatures of a tighter ion-pair. ${ }^{1 c}$ The situation in which the cations reside close to the carbons bearing the highest charge density would lead to a higher degree of localization of charge on the relevant carbons. As the temperature is reduced, the solvation shell can rebuild itself, the anion-cation interaction becomes weaker, thus leading to a more efficient charge delocalization. Such an effect results in a more homogeneous delocalization and the charge is delocalized on remote carbon atoms ( $\mathrm{C}-1$ to $\mathrm{C}-8)$ as well. These carbons are high-field shifted, owing to extra negative charge residing on them as a result of the more efficient delocalization. It should be noted that an effect of ion pairing on electron delocalization was shown by Müllen. ${ }^{7 c}$ The sodium cation is solvated less efficiently than lithium and tends to shift the equilibrium towards the formation of tight ion-pairs. As the proton spectrum is very sensitive to the effects of magnetic anisotropy it is shifted upon elevation of temperature to low field owing to a reduction of its paratropicity as a result of a less efficient delocalization. ${ }^{24}$ Tight ion-pairs tend to concentrate the charge of the $4 n \pi$ system, charge is more localized and the paratropicity, which is linked to delocalization and to a "paramagnetic ring current', is quenched. ${ }^{24}$ This effect of the counter cation can explain the line broadening of the $3^{2-} / 2 \mathrm{Li}^{+}$. The more efficient solvation of lithium (in ether solvents) enables a better delocalization and is therefore more paratropic. At $200 \mathrm{~K}$ 
the centre of gravity of the lithium salt lies at $4.20 \mathrm{ppm}$ while that of the sodium salt lies at $4.40 \mathrm{ppm}$. This higher paratropicity of the lithium salt results from a narrower LUMO-HOMO gap thus facilitating the thermal excitation. Calculations lend strength to our explanation of the behaviour of the lithium $v s$. the sodium salt. When the lithium atoms are removed in an unoptimized calculation from their optimal location (Fig. 6) in $3^{2-} / 2 \mathrm{Li}^{+}$(structure A, Table 3) variations in the LUMOHOMO gap can be seen (Table 4). Removal of the cation, which is analogous to the formation of a higher degree of cation solvation, shows narrowing of the LUMO-HOMO gap. This situation affords a more paratropic system and a more efficient charge delocalization over the entire framework

\section{Experimental}

NMR Spectra. $-{ }^{1} \mathrm{H},{ }^{13} \mathrm{C}$ and all the $2 \mathrm{D}$ NMR spectra were obtained with a Bruker AMX-400 spectrometer operating at $400.13 \mathrm{MHz}$ for proton and $100.62 \mathrm{MHz}$ for carbon with a deuterium lock, equipped with an X-32 computer. All spectra are reported relative to $\mathrm{Me}_{4} \mathrm{Si}$. The ${ }^{1} \mathrm{H}$ and ${ }^{13} \mathrm{C}$ NMR absorptions of THF at $3.57 \mathrm{ppm}$ and at $67.1 \mathrm{ppm}$, respectively, were used for calibration. The assignments were assisted by 2D-NMR programs from Bruker library UXNMR.

Materials.-The product 3 was obtained by a modified literature procedure ${ }^{17}$ and gave satisfactory microanalyses and m.p. It was prepared via a Wittig reaction followed by a photochemical ring-closure reaction.

Wittig Reaction.-(a) Preparation of phosphonium salt. 2-Bromomethyl naphthalene $(0.04 \mathrm{~mol}, 8.5 \mathrm{~g})$ [obtained by standard bromination of 2-methylnaphthalene (Aldrich) by NBS], and triphenyl phosphine $(0.04 \mathrm{~mol}, 10.5 \mathrm{~g})$ were dissolved in $p$-cymene $\left(60 \mathrm{~cm}^{3}\right)$, and refluxed for $2.5 \mathrm{~h}$. The mixture was cooled, filtered and washed with light petroleum. Yield $85 \%$ $\left(0.035 \mathrm{~mol}, 16.9\right.$ g), m.p. $240^{\circ} \mathrm{C} ; \delta_{\mathrm{H}}\left(\mathrm{CDCl}_{3}\right) 7.1-8.0(\mathrm{~m}, 22 \mathrm{H})$, $5.6(\mathrm{~d}, 2 \mathrm{H}) ; m / z 483$.

(b) Preparation of $(\mathrm{E}+\mathrm{Z})$ 1-(2-naphthyl)-2-(9-phenanthryl)ethene. The dry phosphonium salt $(0.01 \mathrm{~mol}, 4.8 \mathrm{~g})$ was dissolved in dry THF $\left(40 \mathrm{~cm}^{3}\right)$ (distilled over potassium). Butyllithium was added $(0.015 \mathrm{~mol})$ under an inert atmosphere and the mixture was stirred until it became red and all the phosphonium salt had dissolved. The mixture was left standing for another $2.0 \mathrm{~h}$. The phenanthrene-9-carbaldehyde (Aldrich) $\left(0.02 \mathrm{~cm}^{3}, 4.1 \mathrm{~g}\right)$ in dry THF $\left(30 \mathrm{~cm}^{3}\right)$ was then added dropwise while the reaction mixture was cooled. The reaction mixture was left standing for a further $12 \mathrm{~h}$. The mixture was extracted with water, the solvent evaporated, and the product was purified by column chromatography on silica gel to give a mixture of $E$ and $Z$ 1-(2-naphthyl)-2-(9-phenanthryl)ethene. Yield $40 \%$ (0.003 mol, 2.6 g). M.p. $140^{\circ} \mathrm{C}$ (lit., ${ }^{17} 143^{\circ} \mathrm{C}$ ). (Found: C, 94.8 ; $\mathrm{H}, 5.55$. Calc. for $\mathrm{C}_{26} \mathrm{H}_{18}: \mathrm{C}, 94.54 ; \mathrm{H}, 5.45 \%$ ).

Photocyclization of $(\mathrm{E}+\mathrm{Z})$ 1-(2-naphthyl)-2-(9-phenanthryl)ethene.-Preparation of 3. A quartz photoreactor with forced water circulation, equipped with an unfiltered $\mathrm{Hg}$ high pressure lamp TQ150 was fitted into a pyrex cell containing the 1-(2-naphthyl)-2-(9-phenanthryl)ethene $\left(7.5 \times 10^{-4} \mathrm{~mol}\right.$, $0.25 \mathrm{~g}$ ) dissolved in $800 \mathrm{~cm}^{3}$ extra pure benzene (Merck) and some crystals of iodine were added. The course of the reaction was followed by TLC on silica gel. When the reaction was completed (after about $4.5 \mathrm{~h}$ ), the benzene solution was washed with saturated aqueous $\mathrm{Na}_{2} \mathrm{~S}_{2} \mathrm{O}_{3}$, dried $\left(\mathrm{Na}_{2} \mathrm{SO}_{4}\right)$ and evaporated. The products were purified and separated from the oily reaction mixture by column chromatography on alumina in the usual way. Elution with benzene-hexane mixtures with increasing solvent ratio, gave three products. (a) Dibenzo $[c, g]$ chrysene, eluted with benzene-hexane $(1: 8)$ Yield $20 \%\left(1.5 \times 10^{-4} \mathrm{~mol}, 50 \mathrm{mg}\right)$, m.p. $117^{\circ} \mathrm{C}$ (lit., $\left.{ }^{17} 178^{\circ} \mathrm{C}\right)$. (Found: C, 95.0; H, 5.15. Calc. for $\mathrm{C}_{26} \mathrm{H}_{16}$ : C, 95.12; H, 4.88\%); $m / z 328$.

(b) Dibenzo[e,ghi]perylene, eluted with benzene, yield $8 \%$ $\left(6 \times 10^{-5} \mathrm{~mol}, 10 \mathrm{mg}\right)$, m.p. $300^{\circ} \mathrm{C}$ (lit., $\left.{ }^{17} 302{ }^{\circ} \mathrm{C}\right) ; \mathrm{m} / \mathrm{z} 326$.

The desired dibenzo $[b, g]$ chrysene 3 was eluted with benzenehexane (1:3) (the middle fraction). Yield $18 \%\left(1.3 \times 10^{-4} \mathrm{~mol}\right.$, $45 \mathrm{mg}$ ); m.p. $210{ }^{\circ} \mathrm{C}$ (lit., ${ }^{17} 212{ }^{\circ} \mathrm{C}$ ). (Found: C, 95.3; H, 4.65 . Calc. for $\left.\mathrm{C}_{26} \mathrm{H}_{16}: \mathrm{C}, 95.12 ; \mathrm{H}, 4.88 \%\right) ; m / z 328 .{ }^{1} \mathrm{H}$ and ${ }^{13} \mathrm{C}$ NMR spectroscopic data are given in Tables 1 and 2.

Preparation of Anions.-Lithium or sodium wire were introduced into the upper part of an extended NMR tube containing the polycyclic compound $(10-20 \mathrm{mg})$ dissolved in $\left[{ }^{2} \mathrm{H}_{8}\right] \mathrm{THF}$ (Aldrich) $\left(0.7 \mathrm{~cm}^{3}\right)$. The frozen solution was degassed and sealed under vacuum. The solution was brought in contact with the alkali metal by inverting the tube. Formation of the anion was detected by ${ }^{1} \mathrm{H}$ NMR spectroscopy. When the NMR study was completed, the solution was quenched with $\mathrm{O}_{2}$. Only the starting material was observed.

MNDO Calculations.-The calculations were performed using the MNDO program ${ }^{19-21}$ modified by Professor A. Goldblum on a VAX 9000 running under VMS operating system program

\section{Acknowledgements}

Financial support from the Basic Research Foundation administered by the Israel Academy of Sciences and Humanities and the Volkswagen-Stiftung, Germany, is gratefully acknowledged. NMR facilities were funded by the Margaret $M$. Thatcher Basic Equipment Laboratory.

\section{References}

1 (a) D. J. Cram, Fundamentals of Carbanion Chemistry, Academic Press, New York, 1963; (b) E. M. Kaiser and O. W. Slocum, in Organic Reactive Intermediates, ed. S. P. McManus, Academic Press, New York, 1973, Ch. 5; (c) M. Szwarc, Ions and Ion Pairs in Organic Reactions, Wiley Interscience, New York, 1972, vol. 1, vol. 2; (d) M. Szwarc, Acc. Chem. Res., 1969, 2, 87; (e) M. Szwarc, Acc. Chem. Res., $1972,5,169$

2 (a) E. Buncel, Carbanions: Mechanistic and Isotope Aspects, Elsevier, Amsterdam, 1945; (b) Comprehensive Carbanion Chemistry, eds. E. Buncel and T. Drust, Elsevier, New York, 1981, Part A

3 (a) J. Thiele, Ber., 1900, 33, 660; (b) W. Schlenk, J. Appenrodt, A. Michael and A. Thal, Chem. Ber., 1914, 47, 473.

4 For recent selected reviews see: (a) A. Krief, Tetrahedron, 1980, 36 2531; (b) J. F. Biellman and J. B. Ducep, Org. React., 1982, 27, 1; (c) B. T. Grobel and D. Seebach, Synthesis, 1977, 357.

5 (a) J. M. Lehn and G. Wipff, J. Am. Chem. Soc., 1976, 98, 708; (b) P. v. R. Schleyer, Pure Appl. Chem., 1984, 56, 151

6 (a) R. G. Harvey, Synthesis, 1970, 4, 161; (b) P. W. Rabideau in Chemistry of Polynuclear Aromatics, ed. L. B. Ebert, ACS Advances in Chemistry, Series No. 217, 1987, p. 73.

7 (a) M. Rabinovitz, Top. Curr. Chem., 1988, 146, 101; (b) M. Rabinovitz and Y. Cohen, Tetrahedron, 1988, 44, 6957; (c) K. Müllen, Chem. Rev., 1984, 84, 603

8 (a) A. Minsky, A. Y. Meyer and M. Rabinovitz, Angew. Chem., Int. Ed. Engl., 1982, 22, 45; (b) A. Minsky, A. Y. Meyer, R. Poupko and M. Rabinovitz, J. Am. Chem. Soc., 1983, 105, 2164

9 (a) A. Minsky, A. Y. Meyer and M. Rabinovitz, Tetrahedron Lett. 1982, 23, 5351; (b) A. Minsky, A. Y. Meyer and M. Rabinovitz, Tetrahedron, $1985,41,785$.

10 A. Minsky, Y. Cohen and M. Rabinovitz, J. Am. Chem. Soc., 1985, 107, 1501

11 Y. Cohen, A. Meyer and M. Rabinovitz, J. Am. Chem. Soc., 1986, 108, 7039.

12 M. Rabinovitz and Y. Cohen, in Chemistry of Polynuclear Aromatics, ed. L. R. Ebert, ACS Advances in Chemistry No. 217, 1987, p. 53 
13 R. Frim, A. Goldblum and M. Rabinovitz, J. Chem. Soc., Perkin Trans. 2, 1992, 267.

14 F. Sondheimer, The Chemical Society Special Publication, No. 21 The Chemical Society, London, 1967, p. 75.

15 Y. Cohen, J. Klein and M. Rabinovitz, J. Am. Chem. Soc., 1988, 110 4634.

16 A. Minsky and M. Rabinovitz, J. Am. Chem. Soc., 1984, 106, 6755.

17 A. H. A. Tinnemans and W. H. Laarhorven, J. Am. Chem. Soc., 1974, 96, 4617.

18 B. Eliasson, U. Edlund and K. Müllen, J. Chem. Soc., Perkin Trans. 2, 1986, 937.

19 M. J. S. Dewar and W. Thiel, J. Am. Chem. Soc., 1977, 99, 4899.
20 W. Thiel, From MNDOC QCPE Catalog No. 438, 1982, V2, 63.

21 P. v. R. Schleyer, E. Kauffman and G. W. Spitznagel, Organometallics, 1986, 5, 79 .

22 A. Sygula, K. Lipkovitz and P. W. Rabideau, J. Am. Chem. Soc, $1987,109,6602$.

23 Y. Cohen, Y. Fraenkel, M. Rabinovitz, P. Felder and F. Gerson, Helv. Chim. Acta, 1990, 73, 2048

24 H. Vogler, Mol. Phys., 1985, 55, 951.

Paper $2 / 02759 \mathrm{C}$

Received 27th May 1992

Accepted 2nd July 1992 